\title{
Crowds and Camera Traps: Genres in Online Citizen Science Projects
}

\author{
Holly K. Rosser \\ University of Nebraska at Omaha \\ hrosser@unomaha.edu
}

\author{
Andrea Wiggins \\ University of Nebraska at Omaha \\ wiggins@unomaha.edu
}

\begin{abstract}
Despite the importance of instruction for effective task completion in crowdsourcing, particularly for scientific work, little attention has been given to the design of instructional materials in crowdsourcing and citizen science. Consequences of inattention to tutorial design are further magnified by the diversity of citizen science volunteers. We use digital genre theory to identify the norms of tutorial design for the most abundant citizen science project type on the Zooniverse platform, camera trap image classification, where a highly-standardized task structure makes it a strong candidate as a specific genre of citizen science.

Comparative content analysis of 14 projects' features, tutorial design, and supporting materials identified a great deal of uniformity in some respects (indicating an emergent genre) but surprising variation in others. As further evidence of an emergent genre, the amount of mentoring the science team received and specific task features of the project appeared to impact tutorial design and supporting resources. Our findings suggest that genre theory provides a useful lens for understanding crowd science projects with otherwise disparate characteristics and identifying instances where the digital medium can be deployed more effectively for task instruction.
\end{abstract}

\section{Introduction}

Despite the increasing popularity of crowdsourcing among some parts of the scientific community, many researchers still have reservations about the quality and utility of crowdsourced research projects [13]. Hesitancy to adopt crowdsourcing for research was based on a variety of concerns including competency issues, which instructional materials and training programs are typically designed to address. To promote effective and efficient task completion and participant engagement in citizen science, science teams commonly provide online tutorials for task instruction and skills training. Use of tutorials for task instruction differentiates citizen science from most crowdsourcing platforms, such as Amazon's Mechanical Turk or CrowdFlower, where instructions are often provided as part of the assignment and not as a stand-alone digital document.

To date, there has been little research on effective design of tutorials to train citizen science volunteers in key scientific skills, such as species identification and image content analysis. Online citizen science platforms such as the Zooniverse, which enables crowdsourced image classification and transcription, have been available for about the last decade. Compared to most crowd work platforms, citizen science task instruction is further complicated by the fact that imposing specific skill requirements on volunteer participants, such as those used for some tasks on platforms like Amazon Mechanical Turk, is often impossible and usually undesirable. We therefore undertook a descriptive study of the tutorial design and use of additional supporting resources available for volunteers to uncover potential trends or norms in instructional design within a specific, yet common, project type found on Zooniverse.

To focus our research, we leveraged digital genre theory to evaluate possible patterns in project design and instructional materials indicative of genres in online citizen science projects, which would support the potential of genre theory for advancing research in crowd science. We selected camera trap image classification projects, which involve the identification of wildlife from images taken by autonomous stationary camera networks deployed in natural environments, as the focus of this work. These projects were selected because the nature of the volunteers' task - identifying wildlife in images - was strikingly similar across a wide variety of projects around the world, in both urban and rural locations, despite the platform allowing and supporting alternate configurations for this task. Examining projects with strong task similarity allowed us to understand how project characteristics interact with variations in tutorial design and other supporting 
materials, which may offer useful insights for future improvements to task instruction in similar projects.

The goal of this study was to evaluate whether there are notable similarities in tutorial design and supporting resource implementation in camera trap image classification projects, which would support the intuitive understanding of these projects as a specific genre based on task design and instructional materials. This approach is aligned with Askehave and Nielsen's [1] description of genre as "communicative purpose realized by move structure realized by rhetorical strategies." The research question was, "Are there project components, such as task characteristics, tutorial design features, and use of supporting resources, that can identify distinct genres of online citizen science projects?"

To examine this question, we evaluated camera trap project features such as specific variations in task type, the amount of mentoring the science teams received during project development, and location type. We identified several key features of tutorial designs to assess, including the number of pages and words, as well as the number and types of images used. We also reviewed key features of projects' supporting resources, including implementation of supplemental resources. We expected to find similarities in the project characteristics and design of supporting resources and tutorials that aligned with specific task types, as in [22], which would provide a reliable way to identify online citizen science project genres. If the trends we expected to observe were present, then digital genre theory could offer a useful tool for research on crowd science.

This paper reviews pertinent literature on digital genre theory, provide background on the Zooniverse, discuss the applicability of genre theory for this research, describe the methods and results of empirical analysis, and discuss implications of the findings for applying digital genre theory in crowd science studies.

\section{Background}

Prior research on digital genre theory provides a novel lens for assessing the instructional materials supporting citizen science participation. We review digital genre theory, the Zooniverse as a platform for a specific genre of citizen science projects, and the expected utility of genre theory for designing citizen science projects and task instruction materials.

\subsection{Digital Genre Theory}

Genre theory is predicated on categorizing documents based on "purpose, form, and content" $[6,12,16]$. Genre "acts as a template of attributes that are regular and can be systematically identified" [6]. Characterizing a genre provides readers with shared knowledge and understanding of what to expect from the text, which can ultimately result in a reduction of cognitive load [6]. Thus, genre theory can describe both the form a document throughout its lifecycle as well as its functional utility for human activities [12].

Prior research has taken genre theory one step further by discussing how to recognize and use, but not necessarily define, a digital genre. Much like traditional genre theory, digital genre provides a "social purpose" within a "conventionalized internal structure" of text as recognized by members of a community $[1,6,16]$. However, it is important to understand that the environment of the Internet has fundamentally changed the way we recognize and interact with an artifact or digital document. Artifacts may have some of the same characteristics as the printed document they replicate, but digital documents require users to navigate differently through the information it contains. Placing artifacts of similar types and common characteristics within a digital genre provides "normative scope" that helps users better understand and judge the relevance of the page, site, or digital document for a purpose [16]. Once an artifact is identified via its common characteristics by users who share an understanding of that genre, their shared knowledge further promotes the validity of that digital genre in other instances $[6,16]$.

There are multiple ways in which digital genre theory can be used to understand types of task instruction and tutorial design for citizen science and other types of crowdsourcing. It was selected as a framework for our analysis because we expected that emergent genres - of crowdsourcing platforms, project types, or instructional materials design - could help identify underlying commonalities that support more effective designs for crowd science at a higher level. More specifically, we expect that this strategy can guide crowdsourcing platforms like Zooniverse to better isolate characteristics and commonalities that identify project genres. Project genres can then guide design improvements for supporting volunteers, shaping activities with "templates, frameworks, and socially agreed-upon constraints for communicating" [6].

\subsection{The Zooniverse}

The Galaxy Zoo project originated in July 2007 to crowdsource image classification for an astronomy project, and quickly expanded to include other projects, re-launching as the Zooniverse citizen science platform in December 2009 [3, 24]. The development of the Zooniverse platform essentially founded a new 
identifiable genre of citizen science projects, referred to as "virtual" or "online" citizen science [21], in which participation tasks are entirely mediated by technologies. The evolution of the Zooniverse as a platform accelerated in 2015, when the Zooniverse Project Builder was released with a set of standardized tools for independently developing and launching online citizen science projects, enabling substantial growth in the scope and diversity of projects hosted on the platform. When the Zooniverse Project Builder was introduced in 2015, there were approximately 42 projects hosted on the site [23]. At the time of data collection for the current study, Zooniverse hosted 91 projects across a variety of scientific disciplines, including astronomy (Comet Hunters), biology (Etch a Cell), ecology (Snapshots at Sea), and humanities (SCOTUS Notes); out of 54 active projects, 23 focused on camera trap classification. Examples of projects sampled in this study include Snapshot Safari, Chicago Wildlife Watch, and Identify New Zealand Animals.

Within the Project Builder toolkit, guidance was offered in instructions, policies, and best practices, but specifics of task workflow design, implementation of supplemental resources, and refinement of their instructional content were left largely up to the project creators [3, 5, 14]. Key suggestions from Project Builder site included "keeping everything short and simple," using help text to provide "visual examples and question-specific guidance," as well as "using images as examples whenever possible." Project Builder also supports creating platform-specific supplemental resources, including FAQs, Field Guides, "About" pages that describe the research and science team members, as well as additional resources and educational materials offered to participants through links to external resources. Assistance from Zooniverse staff is also available to science teams upon request.

To address both the lack of research on task instruction in crowdsourcing and advance our understanding of genres in online citizen science, we examined current practices of tutorial design (see Figure 1) and use of supplemental resources on the Zooniverse platform. Zooniverse was selected due to its popularity, Project Builder functionality that allows individual research teams to construct their own projects and supporting materials, and relative sophistication as an online platform dedicated to crowdsourced science. The Project Builder toolkit and templates also lends itself nicely to digital genre theory since it conventionalizes citizen science for certain data types. Although the variety of related platforms is increasing, Zooniverse is a leading online citizen science platform.

\subsection{Applying Genre Theory to Online Citizen Science}

One source of reluctance to trust crowdsourced science stems from researchers' concerns that volunteers' work may be unreliable, leading to low quality data [13]. Many professional scientists believe some level of expertise is needed for participants to perform scientific tasks, yet numerous publications across a variety of scientific disciplines have demonstrated that well-designed projects and tasks rarely require disciplinary expertise when they provide useful instruction and carefully scoped tasks for

Figure 1. A tutorial page from a Snapshot Safari project that describes navigation between multiple image frames.

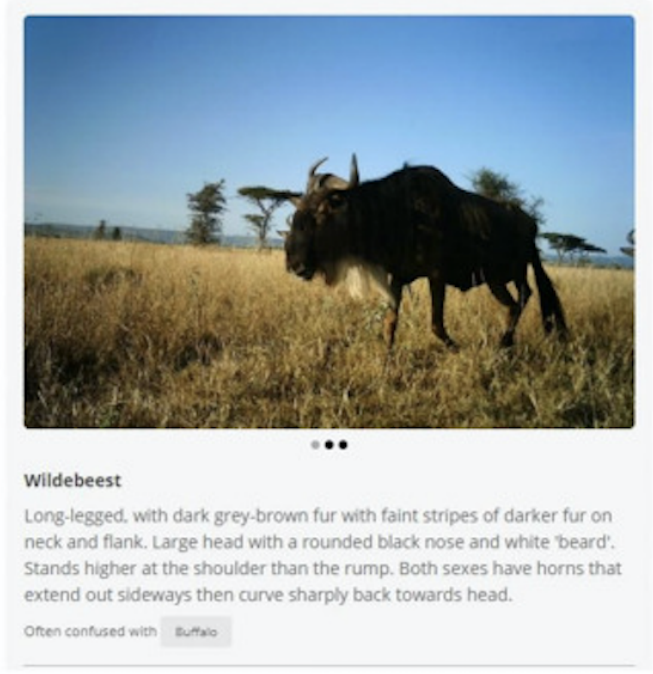

\section{Looks good!}

By comparing the animal to the photos here and reading the description below, we can confirm whether we have the right animal. You can flip through the photos to see examples of the animal from multiple angles using the bullets underneath the picture.

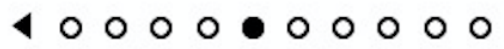


volunteers [11]. A key strategy for achieving reliable results with crowdsourcing is ensuring that appropriate tasks are assigned to volunteers; a common approach is to decompose the research into small simple tasks, with concise instruction for task completion $[4,7,13,18]$. Since tasks are often simplified in ways that are similar across a variety of citizen science projects and platforms (e.g., classifying entities in an image), the instructional materials offered - including tutorials - have potential to take advantage of a genre-based design strategy using templates with a "conventionalized internal structure by which a particular text genre unfolds" [1].

Like any crowdsourcing platform, Zooniverse "has a number of characteristics which significantly contribute to the way the web-mediated genres look and are used" [1]. These include, for example, specific functionalities for task completion, which impact the type of science conducted on the platform and the types of instructional materials that it supports and their presentation. We consider the projects and their supporting materials to be "digital texts" because their presentation and interaction mechanisms are essentially the same as those invoked in digital genre theory. For example, Zooniverse's Project Builder tools allow anyone to quickly and easily set up a project with little effort, for projects focused on classification, identification, counting, drawing, outlining, transcribing, or annotating any type of content found in images, photos, graphs, or texts. With the instructions and guidelines in the Project Builder, a new project can be ready for initial beta testing for approval and promotion to volunteers in as little as 30 minutes [14]. Similar to other crowdsourcing sites, recommendations for Project Builder task designs and supporting resources are limited, and focus primarily on keeping tasks and materials brief and simple [8, 10, 17].

Although existing resources provide very general common sense recommendations for project design, as discussed above, the literature lacks substantive research on the topic of online tutorials, despite a clear need for effective task instruction in online crowdsourcing for scientific work. In the extant literature that discusses task instruction for crowdsource marketplaces in general, the research focus is not specific to task instruction or tutorial design. In addition, successful citizen science projects need to strike a balance between presenting effective task instruction and maintaining participant interest so that the volunteer moves on to completing the research-related tasks requested of them $[4,20]$, which may not necessarily be the case for paid crowdsourcing sites that screen and select participants who are incentivized to task completion with payment. Clearly-written tutorials along with " "quick-start" guides can provide volunteers complementary forms of instruction to better support participants having different levels of familiarity with a given genre of citizen science project [4]. Although tutorials play a key role in initial volunteer contributions, task instruction resources are alternately regarded as either a prompt or barrier to engagement [7]. In practice, Zooniverse staff report many participants initially dismissing tutorials, with some re-opening them later, presumably due to challenges in task completion.

Genres are not currently used to support research and design in citizen science, outside of the development of typologies such as [21], but we believe there is strong potential to leverage this perspective to advance the design of effective online citizen science. The Zooniverse platform currently uses genres in its project listing, displaying eleven scientific disciplines for filtering projects by subject matter. Internally, Zooniverse staff also refer to projects according to scientific discipline or data type in four general categories: Ecology, Biomedical, Astronomy, and Text/Historical Documents. From a design perspective, however, scientific discipline is a suboptimal way to designate project genres, since the same platform features and tools can be used to create similar projects despite differences in scientific discipline. In reality, most data do align with specific task types, and most disciplines align with specific data types, so for the most part, camera trap classification projects are Ecology projects. Nonetheless, a hypothetical social science project on urban blight could use images of urban scenes with an identical task design and supporting resources as the camera trap projects focused on wildlife identification. The methods in this study therefore treat project features, tutorials, and supporting resources as digital texts to evaluate through the lens of genre theory.

\section{Methods}

To examine the task instruction resources provided in the Zooniverse Project Builder, we undertook a comparative content analysis of the tutorials and supporting resources for active projects in early 2018. Using a genre theory lens for guiding this analysis, we purposively sampled and compared a set of projects whose focal data type appears to impose a higher degree of similarity among their task structures and supporting materials, the camera trap image analysis projects. Other Zooniverse project data included still photographs, satellite images, text images, and graphs. Camera trap images were by far the most common data type among Zooniverse projects active in early 2018 and appeared to have strongest uniformity as a genre of Zooniverse projects, providing a suitable focus for our 
Figure 2. A screenshot from a Snapshot Safari project displaying an option grid for classification.



research. The data collection and analysis methods are similar to document analysis techniques [2], which are particularly well suited to genre theory research.

\subsection{Sampling}

The sampling frame started with 55 active workflows in February and March 2018, then reduced to 22 workflows containing single or multi-frame camera trap images for species identification. Of the 22 workflows, five were affiliated with the same organization, Snapshot Safari, with nearly identical workflows and tutorials, so all five Snapshot Safari workflows were averaged together. Two additional workflows were removed from the analysis because they required only a simple binary (yes/no, present/not present) response instead of using an option grid (like the one shown in Figure 2), as is typical for the majority of camera trap projects. The final sample included 14 project workflows, or $25.4 \%$ of the 55 project workflows from the sampling frame.

\subsection{Data collection}

Data were collected primarily through manual content extraction, with participation supporting the interpretation and analysis. Our participation in the projects involved each author in completing classification tasks for all sampled camera trap projects, giving us a thorough understanding of the similarities across workflows and tutorials. We also spoke with Zooniverse platform staff about the process for approval as an official Zooniverse project, and obtained a ranking of the amount of assistance platform staff provided to science teams during the development of each project.

Manual extraction and classification of key characteristics of project tutorials were used for data collection, which involved inductive coding procedures [15]. After developing initial categories of project and tutorial characteristics, we iteratively refined these categories and then comprehensively collected data for the selected sample using manual extraction and content analysis classification based on the refined categories. Excel was used to compile the data as the number of project sampled was limited.

For each tutorial, we tallied the number of pages, count of words, number of images, and number of annotated images or animated GIFs for the tutorial as a whole. Word and image counts provided relevant measures of tutorial design that would be comparable with other citizen science and crowdsourcing platforms. The tally of tutorial pages may be specific to Zooniverse, but offered an alternate measure of tutorial length for this study that may potentially apply elsewhere. As a design feature, these summary statistics represent the relative verbosity and use of supporting visual content, which could relate to differences in the classification task complexity due to project-specific content and additional information in other supplemental resources.

While number of pages might be expected to represent the number of logical actions involved in each task, the similarity of tasks in camera trap projects means that number of pages should show less variance compared to other genres of Zooniverse projects. Generally speaking, more images and less text suggest a more refined tutorial design, with concise, well-developed information. Pages with excessive text may have too much content to be effective at communicating a key point, and more complex tasks may be better supported with more images. Good use of images can potentially replace text, but inserting more than one image per page can affect readability and scrolling. These design principles are likely to apply equally well to other citizen science and crowdsourcing platforms. The Zooniverse platform does not restrict the number of images or amount of text in a tutorial page, nor the number of pages in a tutorial.

To understand the complementary use of supplemental resources, which may reflect on classification task complexity, we calculated the number of species in the option grid provided for selecting responses in each camera trap project (see Figure 2). We also noted whether each workflow had implemented standard platform-supported resource types including FAQs and Field Guides (100\% of sampled projects included tutorials and FAQs), and whether they linked to external resources - specifically blogs and educational resources - in any of their project description materials. There were many instances where projects included external links to partner sites; in most cases, these represented arms-length information that was not specific to the project tasks, 
but more related to marketing, "bigger picture" context, and legitimization of the research. Such sites were not included as providing supplemental information unless they contained a blog specific to the Zooniverse project or contained supplemental materials for educators.

\subsection{Data Analysis}

To analyze the data, we evaluated the mean and standard deviation of the tutorial design features (total page count, total word count, total image count) against the project workflow task features that we expected would influence tutorial design (task type, number of image frames, content focus, option grid size, location types, mentoring level). Mean scores provide a general sense of the usual practices for content design, while standard deviations show design consistency (or inconsistency) across tutorials. Content analysis of categorical data used the procedures described earlier.

Task type was the process or procedure required to complete a task and could include annotation, classification, drawing, outlining, counting, and transcribing, as well as combinations of these tasks. For this study, we focused exclusively on projects with the "classify" task for camera trap images, which also included "classify \& count" tasks. "Classify" tasks involved identifying the type of content in the image, e.g., specific species of African animals in Snapshot Safari projects. "Count" tasks involved tallying entities in images, e.g., number of zebras in Snapshot Safari projects. We also evaluated whether single or multiple frame images were the focus for each task, since multiple frames represents a slightly more complex task. Multiple frame images are a series of three to four images taken in quick succession by motion-triggered camera traps to capture details of moving animals and are viewed individually, which requires learning both how and why to switch between images in the interface.

Tutorial content focus was classified by the instructional style of tutorial content. "Process" tutorials explained the task through a series of written and sometimes itemized instructions, with images used for either descriptive purposes or visual interest, with little instructional value to the images other than identifying project subject matter or interface components within a screenshot. "Example" tutorials explained the task through a series of annotated images and animated GIFs, with few still images or unannotated screenshots (if any), and all images demonstrated tasks. A single "Combined" tutorial explained how to do the task with mixed image types and written instructions describing the process of completing the task.

Mentoring by Zooniverse platform staff was a part of the project development process for some projects, as discussed above. Zooniverse staff provided an ordinal ranking of the amount of direct assistance provided for each sampled project during their development based on internal records, such as email exchanges. "Green" projects were developed entirely without input from Zooniverse platform staff. "Amber" projects had limited support, primarily by email. "Red" projects had substantial support, often as direct collaborators with extensive communication.

Location types had potential to impact the amount of content in tutorials, as it related to the volunteers' assumed familiarity with species in images as well as the number of species at the location. We therefore classified each project based on whether the camera trap network location was rural or urban (defined as a developed area with human residences), and whether it was in North America or other global locations. Urban projects were primarily in North America, and Global projects were primarily in Africa and South America. A notable exception was a project in New Zealand, which was tracking the presence of predators in urban locations, where non-native predators are a primary concern for biodiversity conservation.

\section{Findings}

Descriptive analysis of the project characteristics and tutorial features, shown in Table 1, identified trends in the camera trap projects that may be indicative of genre norms. As expected, we found that the use of images, specifically annotated images and animated GIFs, shortened word count averages in tutorial design, independent of other measures. We also found that most projects took advantage of the FAQ and Field Guide options provided within Project Builder, but there appeared to be trade offs in offering external links to blogs or educational materials. Additionally, we found that the level of mentoring impacted the length, content focus, and use of images of the tutorial. Mentoring also seemed to play a role in the number of supplemental resources provided by a project, particularly external resources. When mentoring was high, tutorial content appeared to be more refined with a greater number of images and fewer words used per tutorial, and provide a more comprehensive set of supporting resources. Finally, location type appeared to play a role in option grid size and, unexpectedly, the number of supplemental resources provided by a project. Although several findings were unsurprising and "as expected", there was no prior empirical evidence to support intuitions about project design, which this analysis has established. 
Table 1. Tutorial measures by project features

\begin{tabular}{l|c|c|c|c|c|c}
\hline Type & $\begin{array}{c}\text { Tutorials } \\
(n, \%)\end{array}$ & $\begin{array}{c}\text { Pages } \\
(\text { mean, sd) }\end{array}$ & $\begin{array}{c}\text { Words } \\
(\text { mean, sd) }\end{array}$ & $\begin{array}{c}\text { Images } \\
(\text { mean, sd) }\end{array}$ & $\begin{array}{c}\text { Annotated/GIFs } \\
(\text { mean, sd) }\end{array}$ & $\begin{array}{c}\text { Option Grid } \\
\text { (mean, sd) }\end{array}$ \\
\hline Classify & $8,50.0 \%$ & $6.6,1.5$ & $374.6,102.8$ & $5.7,1.4$ & $2.1,1.7$ & $38.6,15.3$ \\
Classify \& count & $6,42.8 \%$ & $6.5,2.3$ & $314.8,149.2$ & $6.8,1.5$ & $3.3,2.9$ & $48.7,14.6$ \\
\hline Single frame & $7,50 \%$ & $5.9,1.3$ & $342.3,149.5$ & $6.3,1.4$ & $1.4,1.6$ & $47.4,13.9$ \\
Multi-frame & $7,50 \%$ & $7.3,1.9$ & $373.9,102.8$ & $6.7,2.1$ & $3.8,2.4$ & $38.4,16.3$ \\
\hline Process & $9,64.3 \%$ & $6.3,1.8$ & $359.7,139.6$ & $6.3,1.6$ & $1.5,1.5$ & $46.2,17.3$ \\
Example & $4,28.6 \%$ & $6.3,0.6$ & $286.3,17.4$ & $6.3,0.9$ & $3.5,1.3$ & $39.8,8.1$ \\
Combined & $1,7.1 \%$ & $10.0,-$ & $504.0,-$ & $10.0,-$ & $8.0,-$ & $26.0,-$ \\
\hline Green & $4,28.6 \%$ & $5.5,1.3$ & $362.0,185.1$ & $6.8,1.2$ & $1.0,1.4$ & $49.5,13.7$ \\
Amber & $3,21.4 \%$ & $7.0,2.6$ & $397.7,117.1$ & $4.5,1.4$ & $2.0,1.0$ & $36.7,25.1$ \\
Red & $7,50.0 \%$ & $7.0,1.6$ & $320.7,94.9$ & $6.6,1.3$ & $3.9,2.5$ & $41.9,12.4$ \\
\hline Urban & $4,28.6 \%$ & $6.0,0.8$ & $375.6,126.4$ & $5.3,1.7$ & $2.0,1.4$ & $26.8,9.4$ \\
Rural & $10,71.4 \%$ & $6.8,2.0$ & $338.3,127.3$ & $6.9,1.7$ & $2.9,2.6$ & $49.4,12.1$ \\
\hline North American & $6,42.9 \%$ & $7.0,1.7$ & $384.5,118$ & $7.3,1.4$ & $3.8,2.8$ & $33.7,6.4$ \\
Global & $8,57.1 \%$ & $6.3,1.9$ & $322.4,128.1$ & $5.9,1.9$ & $1.8,1.5$ & $49.9,16.6$ \\
\hline Total & $14,100 \%$ & $6.75,1.1$ & $361.2,51.6$ & $6.5,1.2$ & $2.9,1.8$ & $40.9,8.1$ \\
\hline
\end{tabular}

\subsection{Task type and content focus}

Task type had a moderate impact on tutorial design, with the classify \& count projects including more images on average, and more annotated images and GIFs, compared to classify-only tasks. This was expected: the additional step of counting should require additional instruction. Surprisingly, however, the additional task component did not lead to more tutorial pages or words (although standard deviations were higher for classify \& count tasks), suggesting that additional task instruction was partially accomplished with images instead of text or procedural steps (pages). Slightly more complex tasks also aligned with a higher number of species, but did not lead to longer tutorials.

Single versus multiple frame classification tasks also varied in tutorial design. Despite including fewer species on average, projects with multi-frame images tended to have longer tutorials, corresponding with the slightly more complex task. Multi-frame projects used substantially more annotated and animated images (average 3.8 compared to 1.4), which offered a more straightforward option than text for indicating the interface controls for changing image views.

The content focus of the tutorials was strongly related to tutorial design, partially by definition. Example-oriented tutorials, which were all highly mentored projects, had less text but substantially more use of annotated and animated images than process-oriented projects. Written instructions were short and simple, describing the annotated image or animation rather than the process of completing the task. The majority (7 of 9) of projects with process-oriented tutorials had less mentoring or none; all unmentored projects used a process tutorial. Process-oriented tutorials never used animated GIFs, although animation might seem like a natural tool for demonstrating processes. They were also more text-heavy, relying on words to convey process details instead of images.

The single combined-type tutorial was an outlier on all tutorial features, with more pages, more text, and more images of all types - but surprisingly, the fewest species to classify out of all of the projects in the sample. This tutorial basically "doubled up" its instructional content by describing the process by which the participant completes the task and also explaining the actions taken in the annotated or animated images, although relatively little animation was used. For almost every measure, this tutorial was nearly double the content and length of the other two types. Although this project was highly mentored, the Zooniverse staff noted that its science team did not appear to take design advice as seriously as other projects with similar support.

This exception aside, the more mentoring a project received, the more it used annotated and animated images to explain content. Highly-mentored projects included, on average, twice as many annotated or animated images compared to those with moderate mentoring, and four times as many as unmentored projects. Highly-mentored projects also had the lowest 
average word count, with the lowest standard deviation, and annotated and animated images instead of text.

\subsection{Location type and species diversity}

We evaluated option grid size against location type because we expected to see more grid options (and therefore species to classify) in rural than urban locations, and more species in non-North American locations. This was confirmed, as rural locations tended to contain larger option grids, averaging 48 species, whereas urban sites averaged 26.8 species. As expected, North American projects had fewer species to classify than Global projects, with the exception of New Zealand, which had fewer species in its urban locations.

We also expected that larger option grids would co-occur with longer tutorials and more use of images. In fact, we found that most of the urban projects, which had fewer species on average, had more text on fewer tutorial pages. Use of images was higher for the rural locations than urban ones, as expected, but to our surprise, the projects based in North America (with fewer species) used more images, and more annotated and animated images, than projects outside of North America with higher numbers of species.

\subsection{Mentoring and supplemental resources}

In addition to the prior observations, mentoring was related to use of supplemental resources. More mentoring meant more supplemental resources were implemented, while unmentored projects had fewer supplemental resources.

All of the projects had FAQs and only one had no Field Guide implemented, so this variability was most apparent in external resources. As noted earlier, the two types of external resources, blogs and educational materials, appeared to show a trade-off that suggests limits on the science teams' ability to invest in developing this content. Several projects linked to either a blog or educational materials, but not both, which may suggest other influences playing a role in determining the type of external resources developed for participants.

\subsection{Location type, tutorial design and supporting resources}

Two-thirds of North American projects provided external blogs, and two-thirds provided educational resources. However, non-North American projects had lower rates of external resources (3 of 8 for both types). While we cannot rule out funding or partnership requirements that led North American projects to include more external resources, the higher rates may be a product of the increased mentoring reported by Zooniverse. Four of the six North American projects received a high level of mentoring, one of the six received some mentoring and the remaining project received no mentoring. As with other highly-mentored projects, North American projects used more images, including annotated and GIFs, than their Global counterparts. However, tutorial length measures of average page count and word count were also higher for North American projects, which contradicts other findings for projects with more mentoring. This was also surprising given the average larger option grid size for global projects. One would expect that page count and word count would be higher for global projects with greater species diversity, but this was not the case.

\section{Discussion}

Our analysis identified several relationships between project features, tutorial design, and supplemental resources. Several of these trends were as expected: more complex tasks required a bit more instruction, more species to classify tended to mean more images were used, and images with annotation or animation can replace textual description. However, the apparent influence of mentoring on the development of genre in these projects was strong, in keeping with theory.

\subsection{The Importance of Mentoring}

We expected that mentoring from Zooniverse platform staff might support further refinement in supporting materials, which appeared to be true. These projects appeared to have taken better advantage of the digital medium and substituted images for words in their tutorials, with lower standard deviations in number of words showing that they were more uniformly concise in their communication.

The relationship between mentoring and tutorial style (process or example) was also striking. The fundamental difference in tutorial styles - for essentially the same task across all projects sampled - may reflect the science teams' assumptions about volunteers' familiarity with the basic tasks. If volunteers are assumed to be unfamiliar with identifying species in an image, process-oriented instructions are given; when example-oriented tutorials are provided, they assumed that only project-specific details need to be conveyed. Given the uniformity of these projects' tasks and the fact that most Zooniverse volunteers engage in multiple projects, the latter assumption seems more appropriate and clearly related to a succinct "show-don't-tell" style of tutorial design. If this interpretation is on target, it suggests that one of the biggest benefits of mentorship 
is conveying a sense of "normative scope" regarding volunteers' platform fluency so that instructional efforts are targeted to developing instructional content.

Mentorship also appeared to have a substantial impact on the number of supporting resources that projects provided. Although we can't rule out external influences, we suspect that science teams willing to reach out for assistance and guidance were also more motivated to invest the effort to create materials to enhance the experience for volunteers.

\subsection{Genre Theory for Crowd Science}

A goal of this study was to evaluate the utility of digital genre theory for assessing the design of online citizen science projects and instructional materials supporting participant success. The degree of similarity across the projects suggests that genre theory may have value for research in crowd science beyond the camera-trap classification projects found on the Zooniverse platform. Although there were notable differences, on the whole, the Zooniverse camera trap projects showed a great deal of similarity among themselves that cannot be attributed solely to the platform. Classification and counting are very common microtasks throughout crowdsourcing platforms. Such similar task types suggests some of our results may be suited for comparison to other crowdsourcing contexts.

Despite substantial diversity in the research it supports, the Zooniverse platform's features provide stability and "fixity to otherwise variable texts" [12]. By providing scientists with refined, templated resources to build robust crowdsourced research projects in a consistent and cohesive manner, citizen science platforms help address the problems of reliability that prevent researchers from considering crowdsourced research. Genre-specific functionality also provides a set of tools to improve volunteer experiences and engagement [20], largely through a priori application of research, experience, and best practices that effortlessly propagate the hard-won lessons for successful strategies across a wider range of projects.

Although most researchers would prefer a dedicated pool of trained volunteers [19], volunteers are known to 'dabble' in any number of tasks and projects [7]. Recent research has shown that many citizen science volunteers participate in multiple projects simultaneously [9]. Zooniverse project staff have also reported that volunteers tend to sample new projects when they are introduced but spend most of their time in one or two preferred projects, congruent with the observation that "participation...is inherently non-competitive" [20]. The use of genre-based tools and templates for project design and the development of supporting materials and tutorials, which would require greater similarity across projects, would also be advantageous for participants, supporting easy movement between projects with less cognitive overhead [12].

\section{Conclusions}

This research described current norms in tutorial design and supporting resources for Zooniverse camera trap classification projects and demonstrated the applicability of genre theory to crowdsourcing studies. Future work will examine other task types commonly found in microtasking to further investigate the use of genre as a lens to understand citizen science. Our results represent a snapshot in time, but we believe they can inform the development of genre-based templates and tools for Zooniverse and provides a basis for future work on online task instruction for crowdsourcing more broadly. Direct guidance appeared to have a strong impact on developing and enforcing genre norms in project design and instructional materials, suggesting potential to leverage these findings for improved design for online citizen science across platforms.

\section{Acknowledgements}

This publication uses data generated via the Zooniverse.org platform, development of which is funded by generous support, including a Global Impact Award from Google, and by a grant from the Alfred P. Sloan Foundation.

We thank Grant Miller for providing input on staff involvement with camera trap project development.

This work was supported in part by the Nebraska Research Initiative.

\section{References}

[1] Inger Askehave and Anne Ellerup Nielsen. What are the characteristics of digital genres? genre theory from a multi-modal perspective. In Proceedings of the 38th Annual Hawaii International Conference on System Sciences, pages 98a-98a. IEEE, 2005.

[2] Glenn A Bowen. Document analysis as a qualitative research method. Qualitative research journal, 9(2):27-40, 2009.

[3] Alex Bowyer, Chris Lintott, Greg Hines, Campbell Allan, and Ed Paget. Panoptes, a Project Building Tool for Citizen Science. In Proceedings of the AAAI Conference on Human Computation and 
Crowdsourcing (HCOMP15). AAAI, San Diego, CA, USA, pages 1-2, 2015.

[4] T. Causer and V. Wallace. Building a volunteer community: results and findings from Transcribe Bentham. Digital Humanities Quarterly, 6, 2012.

[5] Kevin Crowston. Gravity spy: Humans, machines and the future of citizen science. In Companion of the 2017 ACM Conference on Computer Supported Cooperative Work and Social Computing, pages 163-166. ACM, 2017.

[6] Kevin Crowston, Barbara Kwaśnik, and Joseph Rubleske. Problems in the use-centered development of a taxonomy of web genres. In Genres on the Web, pages 69-84. Springer, 2010.

[7] Alexandra Eveleigh, Charlene Jennett, Ann Blandford, Philip Brohan, and Anna L. Cox. Designing for dabblers and deterring drop-outs in citizen science. pages 2985-2994. ACM Press, 2014.

[8] Ailbhe Finnerty, Pavel Kucherbaev, Stefano Tranquillini, and Gregorio Convertino. Keep it simple: Reward and task design in crowdsourcing. In Proceedings of the Biannual Conference of the Italian Chapter of SIGCHI, page 14. ACM, 2013.

[9] Catherine Hoffman, Caren B Cooper, Eric B Kennedy, Mahmud Farooque, and Darlene Cavalier. Scistarter 2.0: A digital platform to foster and study sustained engagement in citizen science. In Analyzing the Role of Citizen Science in Modern Research, pages 50-61. IGI Global, 2017.

[10] Aniket Kittur, Jeffrey V Nickerson, Michael Bernstein, Elizabeth Gerber, Aaron Shaw, John Zimmerman, Matt Lease, and John Horton. The future of crowd work. In Proceedings of the 2013 conference on Computer supported cooperative work, pages 1301-1318. ACM, 2013.

[11] Margaret Kosmala, Andrea Wiggins, Alexandra Swanson, and Brooke Simmons. Assessing data quality in citizen science. Frontiers in Ecology and the Environment, 14(10):551-560, 2016.

[12] Barbara H Kwaśnik and Kevin Crowston. Genres of digital documents introduction to the special issue of information, technology \& people.

[13] Edith Law, Krzysztof Z. Gajos, Andrea Wiggins, Mary L. Gray, and Alex Williams. Crowdsourcing as a Tool for Research: Implications of Uncertainty. pages 1544-1561. ACM Press, 2017.
[14] Miller, Grant. A whole new Zooniverse, June 2015.

[15] Kimberly A Neuendorf. The content analysis guidebook. Sage, 2016.

[16] Mark A Rosso. User-based identification of web genres. Journal of the Association for Information Science and Technology, 59(7):1053-1072, 2008.

[17] Thimo Schulze, Stefan Seedorf, David Geiger, Nicolas Kaufmann, and Martin Schader. Exploring task properties in crowdsourcing-an empirical study on mechanical turk. In ECIS, volume 11, pages 1-1, 2011.

[18] James Sprinks, Jessica Wardlaw, Robert Houghton, Steven Bamford, and Jeremy Morley. Task Workflow Design and its impact on performance and volunteers' subjective preference in Virtual Citizen Science. International Journal of Human-Computer Studies, 104:50-63, August 2017.

[19] Ellinore J Theobald, Ailene K Ettinger, Hillary K Burgess, Lauren B DeBey, Natalie R Schmidt, Halley E Froehlich, Christian Wagner, Janneke HilleRisLambers, Joshua Tewksbury, MA Harsch, et al. Global change and local solutions: Tapping the unrealized potential of citizen science for biodiversity research. Biological Conservation, 181:236-244, 2015.

[20] Ramine Tinati, Max Van Kleek, Elena Simperl, Markus Luczak-Rsch, Robert Simpson, and Nigel Shadbolt. Designing for Citizen Data Analysis: A Cross-Sectional Case Study of a Multi-Domain Citizen Science Platform. pages 4069-4078. ACM Press, 2015.

[21] Andrea Wiggins and Kevin Crowston. From conservation to crowdsourcing: A typology of citizen science. In 44th Hawaii international conference on System Sciences, pages 1-10. IEEE, 2011.

[22] Andrea Wiggins and Kevin Crowston. Goals and tasks: Two typologies of citizen science projects. In 45th Hawaii International Conference on System Science (HICSS), pages 3426-3435. IEEE, 2012.

[23] Poonam Yadav and John Darlington. Design Guidelines for the User-Centred Collaborative Citizen Science Platforms. arXiv:1605.00910 [cs], May 2016. arXiv: 1605.00910.

[24] The Zooniverse. The Zooniverse is Go., December 2009. 